\title{
Phase II Study of the Efficacy and Safety of Pembrolizumab for Relapsed/Refractory Classic Hodgkin Lymphoma
}

Robert Chen, Pier Luigi Zinzani, Michelle A. Fanale, Philippe Armand, Nathalie A. Johnson, Pauline Brice, John Radford, Vincent Ribrag, Daniel Molin, Theodoros P. Vassilakopoulos, Akihiro Tomita, Bastian von Tresckow, Margaret A. Shipp, Yinghua Zhang, Alejandro D. Ricart, Arun Balakumaran, Craig H. Moskowitz, for the KEYNOTE-087 Investigators

Author affiliations and support information (if applicable) appear at the end of this article.

Published at jco.org on April 25, 2017.

Processed as a Rapid Communication manuscript.

A complete list of investigators in the KEYNOTE-087 trial is provided in the Data Supplement.

Clinical trial information: NCT02453594.

Corresponding author: Craig $\mathrm{H}$.

Moskowitz, MD, Memorial Sloan Kettering Cancer Center, 1275 York Ave, New York, NY 10065; e-mail: moskowic@ mskcc.org.

(C) 2017 by American Society of Clinical Oncology

0732-183X/17/3519w-2125w/\$20.00
ASSOCIATED CONTENT

Q

Data Supplements

DOI: https://doi.org/10.1200/JCO 2016.72.1316

DOI: https://doi.org/10.1200/JCO.2016. 72.1316

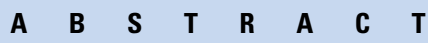

\section{Purpose}

Hodgkin Reed-Sternberg cells harbor alterations in chromosome 9p24.1, leading to overexpression of programmed death-ligand 1 (PD-L1) and PD-L2. Pembrolizumab, a programmed death 1-blocking antibody, demonstrated a high overall response rate (ORR) in patients with relapsed or refractory classic Hodgkin lymphoma ( $r \mathrm{rHL}$ ) in phase I testing.

\section{Methods}

KEYNOTE-087 (ClinicalTrials.gov identifier, NCT02453594) was a single-arm phase II study of pembrolizumab in three cohorts of patients with $\mathrm{rHH}$, defined on the basis of lymphoma progression after (1) autologous stem cell transplantation (ASCT) and subsequent brentuximab vedotin (BV); (2) salvage chemotherapy and BV, and thus, ineligible for ASCT because of chemoresistant disease; and (3) ASCT, but without BV after transplantation. Patients received pembrolizumab $200 \mathrm{mg}$ once every 3 weeks. Response was assessed every 12 weeks. The primary end points were ORR by central review and safety.

\section{Results}

A total of 210 patients were enrolled and treated (69 in cohort 1, 81 in cohort 2, and 60 in cohort 3). At the time of analysis, patients received a median of 13 treatment cycles. Per central review, the ORR was $69.0 \%(95 \% \mathrm{Cl}, 62.3 \%$ to $75.2 \%)$, and the complete response rate was $22.4 \%$ (95\% $\mathrm{Cl}, 16.9 \%$ to $28.6 \%$ ). By cohort, ORRs were $73.9 \%$ for cohort 1, 64.2\% for cohort 2, and $70.0 \%$ for cohort 3. Thirty-one patients had a response $\geq 6$ months. The safety profile was largely consistent with previous pembrolizumab studies.

\section{Conclusion}

Pembrolizumab was associated with high response rates and an acceptable safety profile in patients with $\mathrm{rHH}$, offering a new treatment paradigm for this disease.

\section{J Clin Oncol 35:2125-2132. (C) 2017 by American Society of Clinical Oncology}

\section{INTRODUCTION}

Classic Hodgkin lymphoma (cHL) is a highly curable malignancy with conventional chemotherapy or chemoradiotherapy, but treatment is suboptimal for relapsed or refractory $\mathrm{cHL}(\mathrm{rrHL}) .{ }^{1,2}$ The standard of care for patients with rrHL is salvage chemotherapy, followed by autologous stem cell transplantation (ASCT) if the disease is chemosensitive. ${ }^{3,4}$ Brentuximab vedotin (BV) is indicated after failure of these therapies and was recently approved as consolidation treatment after ASCT in patients at high risk for relapse. ${ }^{5,6}$ Although BV demonstrates an overall response rate
(ORR) of 75\% after ASCT failure, ${ }^{7}$ median duration of response (DOR) is only 6.7 months. In a retrospective analysis of two phase I studies with 20 transplantation-naive patients, 18 of whom refused or were ineligible for ASCT because of chemoresistant disease, the response rate of $\mathrm{BV}$ was $30 \%{ }^{8}$

$\mathrm{cHL}$ is characterized by malignant Hodgkin Reed-Sternberg (HRS) cells dispersed within an extensive inflammatory/immune cell infiltrate. ${ }^{9,10}$ HRS cells frequently harbor alterations in chromosome 9p24.1, leading to overexpression of programmed death-ligand 1 (PD-L1) and PD-L2, ligands of the programmed death 1 (PD-1) immune checkpoint receptor. ${ }^{11,12}$ rrHL may thus be genetically susceptible to blockade of the PD-1 pathway. 
Pembrolizumab is a highly selective, humanized monoclonal immunoglobulin $\mathrm{G} 4 / \kappa$ antibody that blocks the interaction between PD-1 and its ligands; it has shown robust antitumor activity and a favorable safety profile and is approved in multiple tumor types. ${ }^{13,14}$ A flat exposure-response relationship has been found in the dose range of 2 to $10 \mathrm{mg} / \mathrm{kg}$ across clinical studies, ${ }^{15}$ and on the basis of population pharmacokinetic models, the fixed dose of pembrolizumab $200 \mathrm{mg}$ once every 3 weeks is within this range.

In a phase Ib trial (ClinicalTrials.gov identifier, NCT01953692), pembrolizumab demonstrated an ORR of $65 \%$ in patients with heavily pretreated rrHL. ${ }^{16}$ Because of the high unmet need for improved treatments for patients with $\mathrm{rrHL}$ in whom ASCT and subsequent therapies failed or who are ineligible for transplantation, a phase II study was designed to evaluate the clinical activity of pembrolizumab in three separate cohorts, representing the spectrum of relapsed or refractory disease with varying degrees of prior therapies and transplantation status. Efficacy and safety results from all three cohorts are presented.

\section{METHODS}

\section{Patients}

KEYNOTE-087 (ClinicalTrials.gov identifier, NCT02453594) is a multicenter, single-arm phase II study of pembrolizumab in three cohorts of patients with rrHL. Cohorts were defined based on lymphoma progression after (1) ASCT and subsequent BV; (2) salvage chemotherapy and $\mathrm{BV}$, and thus ineligible for ASCT because of chemoresistant disease; and (3) ASCT but had not received BV after transplantation. Patients in cohort 3 could have received BV as part of primary treatment or as salvage treatment or could have been BV naive. The multicohort design allowed contrast in clinical activity among the three main subgroups of patients, defined according to the permutation of relevant previous therapies.

Eligibility criteria for all cohorts included age $\geq 18$ years, measurable disease, Eastern Cooperative Oncology Group performance status of 0 or 1 , and adequate organ function (hematologic, renal, hepatic, coagulation), as determined by laboratory testing within 7 days of first pembrolizumab dose. Exclusion criteria included diagnosis of immunosuppression or receipt of immunosuppressive therapy within 7 days before first study dose; treatment with a monoclonal antibody within 4 weeks before first study dose; prior chemotherapy, targeted small molecule therapy, or radiation therapy within 2 weeks before first study dose; prior allogeneic hematopoietic stem cell transplantation within the past 5 years; known clinically active CNS involvement; active autoimmune disease requiring systemic treatment in past 2 years; active, noninfectious pneumonitis; prior therapy targeting $\mathrm{T}$-cell costimulation or checkpoint pathways; and known HIV or active hepatitis B or C infection.

All patients provided written informed consent. The study protocol (No. MK-3475-087-02) and all amendments were approved by the independent institutional review boards or ethics committees for each study site and conducted in accordance with the Declaration of Helsinki and the International Conference on Harmonization Guidelines for Good Clinical Practice.

\section{Study Design and Treatment}

Patients received pembrolizumab $200 \mathrm{mg}$ intravenously every 3 weeks without premedication for a maximum of 24 months or until documented confirmed disease progression, intolerable toxicity, or investigator decision. Patients attaining a complete remission (CR) could consider stopping pembrolizumab after a minimum of 6 months of treatment, with $\geq 2$ doses received after documented CR. Continuation of treatment beyond the first assessment of progressive disease (PD) was permitted if the patient was clinically stable and agreed on by the investigator and sponsor.
Response was assessed by computed tomography every 12 weeks according to the Revised Response Criteria for Malignant Lymphomas (RRC; Data Supplement). ${ }^{17}$ Positron emission tomography was performed at weeks 12 and 24 to confirm CR or PD and as clinically indicated.

\section{End Points and Assessments}

Primary end points were ORR by blinded independent central review (BICR) according to RRC and safety. Secondary end points were ORR by investigator review according to RRC; complete remission rate (CRR) by $\mathrm{BICR}$ and investigator assessment according to RRC; progression-free survival (PFS) and DOR by BICR and investigator assessment according to RRC; and overall survival (OS). For assessment of CR, a posttreatment residual mass of any size was permitted if it was negative on positron emission tomography imaging.

Safety was assessed descriptively by monitoring all adverse events (AEs), treatment-related AEs, immune-related AEs, and serious and fatal AEs. AEs were graded according to the National Cancer Institute Common Terminology Criteria for Adverse Events (version 4.0). Patient-reported outcomes were an exploratory end point assessed every cycle for the first 5 cycles, then every 12 weeks, with the EQ-5D questionnaire followed by the European Organization for Research and Treatment of Cancer Quality-of-Life Questionnaire-Core 30 (Data Supplement).

\section{Statistical Analyses}

Efficacy and safety were assessed in all patients who received $\geq 1$ dose of pembrolizumab. Relapsed disease was defined as disease progression after response to the most recent therapy; refractory cHL was defined as failure to achieve CR or partial response to the most recent therapy. ORR was defined as the proportion of patients who achieved CR or partial response using RRC criteria ${ }^{17}$ at any time during the study. Best overall response was defined as best ORR during the period between the first dose and the first documented PD, death, or, in the absence of PD, last efficacy assessment before subsequent therapy. DOR was defined as the time between the first response and the date of the first documented PD, death, or, in the absence of PD, last disease assessment.

ORR and CRR were assessed for all patients and by cohort, using the point estimate and $95 \%$ two-sided exact CI; ORR was calculated using the Clopper-Pearson method. An exact binomial test was conducted versus a fixed control rate for each cohort. The nonparametric Kaplan-Meier method was used to estimate PFS and OS curves and rates. Kaplan-Meier estimates were used for analysis of DOR. A prespecified interim analysis, on the basis of investigator-assessed response, was performed after 30 patients reached the first response assessment in all cohorts.

With 60 patients per cohort in the primary analysis population, the design had $\geq 93 \%$ power (one-sided $2.5 \%$ alpha) to detect an ORR of $\geq 35 \%$ in cohorts 1 and 3 compared with a fixed control rate of $15 \%$ and an ORR of $\geq 20 \%$ in cohort 2 compared with a fixed control rate of $5 \%$, using the exact binomial test (nQuery, Version 2.0 software; Statsols, Cork, Ireland). Additional exploratory subgroup analyses of ORR on the basis of previous lines of therapy and by relapsed or refractory status were conducted across cohorts.

\section{Biomarker Assessment}

$\mathrm{PD}$-L1 expression was determined as previously described, ${ }^{16}$ using fresh or archival formalin-fixed, paraffin-embedded pretreatment tissue sectioned at 4 to 5 microns, with a proprietary immunohistochemical assay developed at QualTek Molecular Laboratories (Newtown, PA) in collaboration with Merck (Kenilworth, NJ). PD-L1 expression was scored by a board-certified pathologist. Three scores were reported separately: intensity score (0 to 3 ), membrane staining score (percentage of tumor cells with membrane staining; $0 \%,>0$ to $<50 \%$, $\geq 50$ to $<100 \%$, or $100 \%$ ), 
and histiocyte score (1 to 3; semiquantitative assessment of histiocytes/ macrophages staining positive; Data Supplement). Histiocyte staining was delineated from HRS cell staining by cytomorphologic assessment by the pathologist.

\section{Role of the Funding Source}

This study was designed by representatives of the study sponsor, Merck, and by academic advisors. The principal investigators and the sponsor were responsible for study oversight. The sponsor was responsible for the collection and maintenance of data. The first and last authors and authors from the study sponsor wrote the first draft of the manuscript with the support of a medical writing team funded by the sponsor; medical writing and editorial assistance was provided by the ApotheCom oncology team (Yardley, PA). All authors participated in reviewing and editing the manuscript, approved the submitted draft, had full access to the data used to write the manuscript and vouched for their accuracy, and attested that the study was conducted in accordance with the protocol.

\section{RESULTS}

\section{Patients and Treatment}

A total of 210 patients were enrolled and treated (69 in cohort 1,81 in cohort 2, and 60 in cohort 3 ) between June 26, 2015, and March 21, 2016, at 51 study sites (Data Supplement). As of the September 25, 2016, data cutoff, 90 patients discontinued and 120 were still receiving study treatment (Fig 1). Median exposure to pembrolizumab was 8.3 months (range, 0.03 to 14.99 months), and median duration of follow-up was 10.1 months (range, 1.0 to 15.0 months). At the time of analysis, patients received a median of 13 treatment cycles (range, 1 to 21 in cohorts 1 and 2, and 3-21 in cohort 3). Baseline characteristics are listed in Table 1. Median age was 35 years (range, 18 to 76 years), and patients received a median of four previous lines of therapy (range, 1 to 12 ), with $86.7 \%$ of patients having received at least three previous lines. By design, all patients in cohorts 1 and 2 had experienced prior BV treatment failure, and in cohort 3,41.7\% of patients had received BV treatment before ASCT.

\section{Clinical Activity}

Rates of response. Per BICR, the ORR across all cohorts was $69.0 \%$ (95\% CI, $62.3 \%$ to $75.2 \%$ ) and the CRR was $22.4 \%$ (95\% CI, $16.9 \%$ to $28.6 \%$; Table 2 ). For the protocol-prespecified primary analysis by cohort per BICR, the ORR was $73.9 \%$ (95\% CI, $61.9 \%$ to $83.7 \%$ ) for cohort $1,64.2 \%$ (95\% CI, $52.8 \%$ to $74.6 \%$ ) for cohort 2 , and $70.0 \%$ (95\% CI, $56.8 \%$ to $81.2 \%$ ) for cohort 3 (Table 2). For each cohort, the protocol H0 hypothesis $(P \leq .20 \vee P>.20)$ was rejected $(P<.001)$. ORR by investigator review was similar to ORR by BICR (Data Supplement). Across all cohorts, $>90 \%$ of patients experienced a decrease in tumor burden (Fig 2), and most responses were observed at the first assessment (Data Supplement).

\section{Additional Subgroup Analysis}

Rates of responses on the basis of prior lines of therapy. ORRs were similar between patients who received $<3$ prior lines of therapy versus those who received $\geq 3$ lines (71.4\% v 68.7\%; Data Supplement).

Rates of response in relapsed or refractory subgroups across cohorts. In an analysis of the 73 patients who were refractory to first-line therapy (primary refractory, $\mathrm{n}=73$ ), ORR was $79.5 \%$ (95\% CI, $68.4 \%$ to $88.0 \%$ ), which was higher than the ORR in patients with disease refractory to front-line therapy, salvage therapy, and BV (cohort 2 ORR, 64.2\%; 95\% CI, 52.8\% to $74.6 \%$; Data Supplement). In addition, a high ORR was reported in patients who were refractory to all previous lines of therapy $(n=23)$, with an ORR of $56.5 \%$ (95\% CI, 34.5\% to 76.8\%; Data Supplement). The ORR in patients who had not previously received BV ( $\mathrm{n}=35$ ) was $71.4 \%$ (95\% CI, $53.7 \%$ to $85.4 \%$; Data Supplement).

For patients whose lymphoma relapsed after three or more prior lines of therapy $(\mathrm{n}=146)$ and in patients with rrHL refractory to at least one previous line $(\mathrm{n}=170)$, ORRs were $67.8 \%$ (95\% CI, $59.6 \%$ to $75.3 \%$ ) and $71.2 \%$ (95\% CI, 63.7\% to $77.9 \%$ ), respectively (Data Supplement). These subgroups were not mutually exclusive, with all 210 patients falling under at least one of these two categories. Fourteen patients in this study went on to receive a stem cell transplantation: 10 allogeneic and four autologous.

\begin{tabular}{|c|c|c|c|c|c|}
\hline \multicolumn{2}{|c|}{ Cohort 1} & \multicolumn{2}{|c|}{ Cohort 2} & \multicolumn{2}{|c|}{ Cohort 3} \\
\hline \multicolumn{2}{|c|}{69 patients started treatment } & \multicolumn{2}{|c|}{81 patients started treatment } & \multicolumn{2}{|c|}{60 patients started treatment } \\
\hline $\begin{array}{l}\text { Completed } \\
\qquad(\mathrm{n}=0)\end{array}$ & $\begin{array}{l}\text { Treatment ongoing } \\
\qquad(\mathrm{n}=43 ; 62.3 \%)\end{array}$ & $\begin{array}{l}\text { Completed } \\
\qquad(\mathrm{n}=0)\end{array}$ & $\begin{array}{l}\text { Treatment ongoing } \\
\qquad(\mathrm{n}=36 ; 44.4 \%)\end{array}$ & $\begin{array}{l}\text { Completed } \\
\qquad(\mathrm{n}=0)\end{array}$ & $\begin{array}{l}\text { Treatment ongoing } \\
\qquad(\mathrm{n}=41 ; 68.3 \%)\end{array}$ \\
\hline Discontinued & $(n=26 ; 37.7 \%)$ & Discontinued & $(n=45 ; 55.6 \%)$ & Discontinued & $(n=19 ; 31.7 \%)$ \\
\hline Adverse event & $(n=4 ; 5.8 \%)$ & Adverse event & $(n=3 ; 3.7 \%)$ & Adverse event & $(n=2 ; 3.3 \%)$ \\
\hline Bone marrow transplant & $(n=1 ; 1.4 \%)$ & Bone marrow transplant & $(n=2 ; 2.5 \%)$ & Bone marrow transplant & $(n=0)$ \\
\hline Clinical progression & $(n=2 ; 2.9 \%)$ & Clinical progression & $(\mathrm{n}=1 ; 1.2 \%)$ & Clinical progression & $(n=1 ; 1.7 \%)$ \\
\hline Complete response & $(n=5 ; 7.2 \%)$ & Complete response & $(n=7 ; 8.6 \%)$ & Complete response & $(n=1 ; 1.7 \%)$ \\
\hline Death & $(\mathrm{n}=1 ; 1.4 \%)$ & Death & $(\mathrm{n}=1 ; 1.2 \%)$ & Death & $(n=0)$ \\
\hline Lost to follow-up & $(n=0)$ & Lost to follow-up & $(\mathrm{n}=1 ; 1.2 \%)$ & Lost to follow-up & $(n=0)$ \\
\hline Physician decision & $(n=3 ; 4.3 \%)$ & Physician decision & $(n=7 ; 8.6 \%)$ & Physician decision & $(n=2 ; 3.3 \%)$ \\
\hline Progressive disease & $(n=10 ; 14.5 \%)$ & Progressive disease & $(n=20 ; 24.7 \%)$ & Progressive disease & $(n=13 ; 21.7 \%)$ \\
\hline Patient withdrawal & $(n=0)$ & Patient withdrawal & $(n=3 ; 3.7 \%)$ & Patient withdrawal & $(n=0)$ \\
\hline
\end{tabular}

Fig 1. Patient disposition. 


\begin{tabular}{|c|c|c|c|c|}
\hline Characteristic & $\begin{array}{c}\text { Cohort } 1(n=69)^{*} \\
\text { After ASCT/BV }\end{array}$ & $\begin{array}{c}\text { Cohort } 2(\mathrm{n}=81)^{*} \\
\text { Ineligible for ASCT } \\
\text { and Experienced } \\
\text { Treatment Failure } \\
\text { With BV }\end{array}$ & $\begin{array}{l}\text { Cohort } 3(n=60)^{*} \\
\text { No BV After ASCT }\end{array}$ & $\begin{array}{l}\text { All Patients } \\
(N=210)\end{array}$ \\
\hline Age, years, median (range) & $34(19-64)$ & $40(20-76)$ & $32(18-73)$ & $35(18-76)$ \\
\hline Age $\geq 65$ years, No. (\%) & 0 & 15 (18.5) & $3(5.0)$ & $18(8.6)$ \\
\hline \multicolumn{5}{|l|}{ Sex, No. (\%) } \\
\hline Male & $36(52.2)$ & $43(53.1)$ & $34(56.7)$ & $113(53.8)$ \\
\hline Female & $33(47.8)$ & 38 (46.9) & 26 (43.3) & $97(46.2)$ \\
\hline \multicolumn{5}{|l|}{ ECOG performance status, No. (\%) } \\
\hline 0 & $29(42)$ & $44(54.3)$ & 29 (48.3) & $102(48.6)$ \\
\hline 1 & 39 (56.5) & $37(45.7)$ & $31(51.7)$ & $107(51.0)$ \\
\hline 2 & $1(1.4) \dagger$ & $0(0)$ & $0(0)$ & $1(0.5)$ \\
\hline Median lines of systemic therapy (range), No. (\%) & $4(2-12)$ & $4(1-11)$ & $3(2-10)$ & $4(1-12)$ \\
\hline \multicolumn{5}{|l|}{ Prior lines of therapy, No. (\%) } \\
\hline$\geq 3$ & 68 (98.6) & 78 (96.3) & $36(60.0)$ & $182(86.7)$ \\
\hline$<3$ & $1(1.4)$ & $3(3.7)$ & $24(40.0)$ & 28 (13.3) \\
\hline $\begin{array}{l}\text { Refractory disease or relapsed after } \geq 3 \text { lines of } \\
\text { therapy, No. }(\%)\end{array}$ & $69(100)$ & $81(100)$ & $60(100)$ & $210(100)$ \\
\hline Prior radiation therapy, №. (\%) & $31(44.9)$ & $21(25.9)$ & $24(40.0)$ & $76(36.2)$ \\
\hline Bulky lymphadenopathy, №. (\%)‡ & $5(7.2)$ & $11(13.6)$ & $3(5.0)$ & $19(9.0)$ \\
\hline B symptoms at baseline, No. $(\%) \S$ & 22 (31.9) & $26(32.1)$ & 19 (31.7) & 67 (31.9) \\
\hline Previous BV therapy, №. (\%) & $69(100)$ & $81(100)$ & $25(41.7) \|$ & $175(83.3)$ \\
\hline \multicolumn{5}{|c|}{$\begin{array}{l}\text { Abbreviations: ASCT, autologous stem cell transplantation; BV, brentuximab vedotin, ECOG, Eastern Cooperative Oncology Group. } \\
{ }^{*} \text { Cohort } 1 \text { : experienced ASCT and subsequent BV therapy failure. Cohort 2: experienced salvage chemotherapy failure; ineligible for ASCT and experienced BV therapy } \\
\text { failure. Cohort } 3 \text {. experienced ASCT failure and did not receive BV after ASCT. } \\
\text { †The patient had ECOG performance status of } 1 \text { at screening and ECOG performance status of } 2 \text { at cycle } 1 \text {, day } 1 \text {. } \\
\text { \#Bulky disease was defined as a mass larger than one third of transthoracic diameter at any level of thoracic vertebrae or single site of disease in any area that was } \geq 10 \\
\mathrm{~cm} \text { in diameter. } \\
\S B \text { symptoms include unexplained weight loss of }>10 \% \text { in the past } 6 \text { months; unexplained, persistent, or recurrent fever with temperatures }>38^{\circ} \mathrm{C} \text { during the previous } \\
\text { month; or recurrent drenching night sweats during the previous month. } \\
\| \text { Patients received BV therapy before transplantation. }\end{array}$} \\
\hline
\end{tabular}

Duration of response. A Kaplan-Meier plot of DOR is presented in Figure 2A. Median DOR was not reached in all cohorts (Figs 2B, 2C, and 2D). At 6 months, the OS rate was $99.5 \%$, and the PFS rate was $72.4 \%$. Thirty-one patients $(75.6 \%)$ had a response $\geq 6$ months. Median OS was not reached, with only four deaths occurring. With 97 (46.2\%) patients still receiving pembrolizumab since the start of the study, an ad hoc analysis using a data cutoff of December 31, 2016, was performed and determined that the median OS continued not to be reached, and the 9-month OS and PFS rates were $97.5 \%$ and $63.4 \%$, respectively.

Patient-reported outcomes. The majority of patients experienced maintenance and/or improvement in disease-related symptoms, functioning, and health status, particularly among patients who responded to pembrolizumab (Data Supplement). There was a net improvement in the European Organization for Research and Treatment of Cancer Quality-of-Life Questionnaire-Core 30 global health status/quality of life score and EQ-5D visual analog and utility scores from baseline to week 12 across all cohorts (Data Supplement).

\section{Biomarker Analysis}

At the September 25, 2016, data cutoff, 177 patients had evaluable pretreatment tumor tissue (archival or obtained for study) for immunohistochemistry and best evaluable response per

\begin{tabular}{|c|c|c|c|c|c|c|c|c|}
\hline \multirow[b]{2}{*}{ Response } & \multicolumn{2}{|c|}{$\begin{array}{c}\text { Cohort } 1(n=69) \\
\text { After ASCT/BV }\end{array}$} & \multicolumn{2}{|c|}{$\begin{array}{l}\text { Cohort } 2(n=81) \text { Ineligible } \\
\text { for ASCT and Experienced } \\
\text { Treatment Failure With BV }\end{array}$} & \multicolumn{2}{|c|}{$\begin{array}{c}\text { Cohort } 3(n=60) \text { No BV } \\
\text { After ASCT }\end{array}$} & \multicolumn{2}{|c|}{ All Patients $(N=210)$} \\
\hline & No. (\%) & $95 \% \mathrm{Cl} \dagger$ & No. (\%) & $95 \% \mathrm{Cl}+$ & No. (\%) & 95\% Cl† & No. $(\%)$ & $95 \% \mathrm{Cl} \dagger$ \\
\hline Overall response rate & 51 (73.9) & 61.9 to 83.7 & $52(64.2)$ & 52.8 to 74.6 & $42(70.0)$ & 56.8 to 81.2 & $145(69.0)$ & 62.3 to 75.2 \\
\hline Complete remission* & $15(21.7)$ & 12.7 to 33.3 & $20(24.7)$ & 15.8 to 35.5 & $12(20.0)$ & 10.8 to 32.3 & 47 (22.4) & 16.9 to 28.6 \\
\hline Partial remission & $36(52.2)$ & 39.8 to 64.4 & 32 (39.5) & 28.8 to 51.0 & $30(50.0)$ & 36.8 to 63.2 & $98(46.7)$ & 39.8 to 53.7 \\
\hline Stable disease & $11(15.9)$ & 8.2 to 26.7 & $10(12.3)$ & 6.1 to 21.5 & $10(16.7)$ & 8.3 to 28.5 & $31(14.8)$ & 10.3 to 20.3 \\
\hline Progressive disease & $5(7.2)$ & 2.4 to 16.1 & $17(21.0)$ & 12.7 to 31.5 & 8 (13.3) & 5.9 to 24.6 & 30 (14.3) & 9.9 to 19.8 \\
\hline Unable to determine & $2(2.9)$ & 0.4 to 10.1 & $2(2.5)$ & 0.3 to 8.6 & $0(0)$ & - & $4(1.9)$ & 0.5 to 4.8 \\
\hline
\end{tabular}




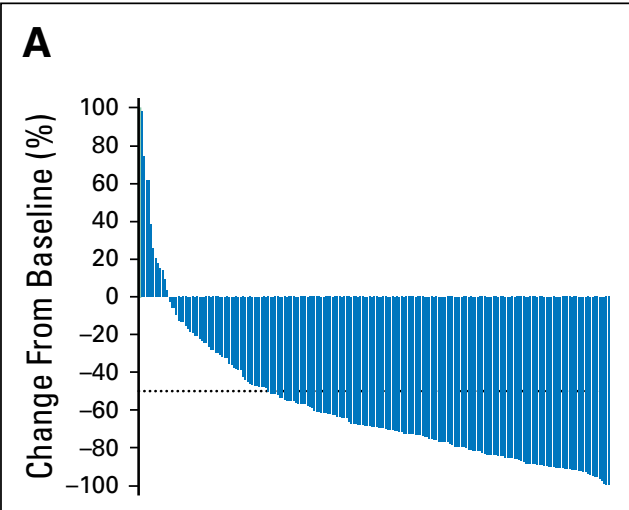

B

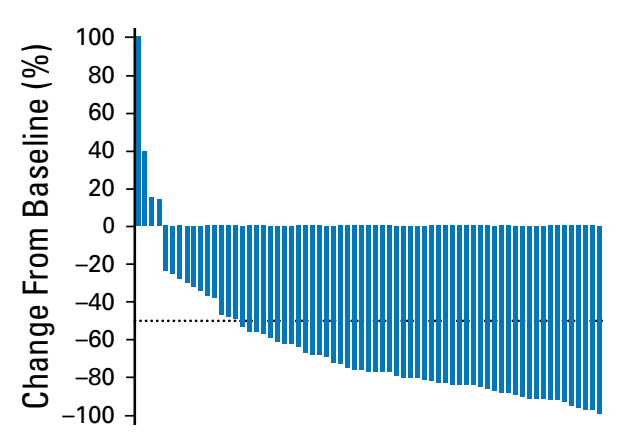

C

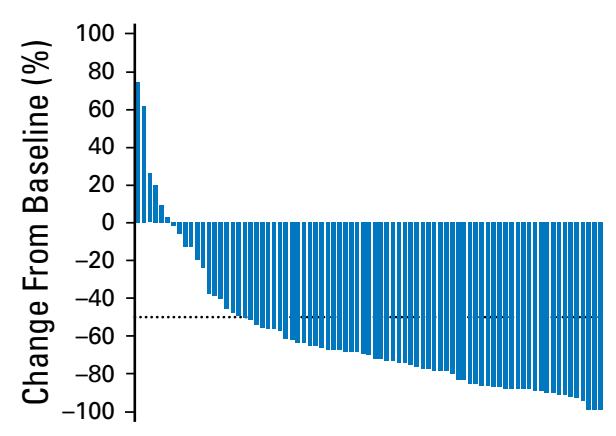

D

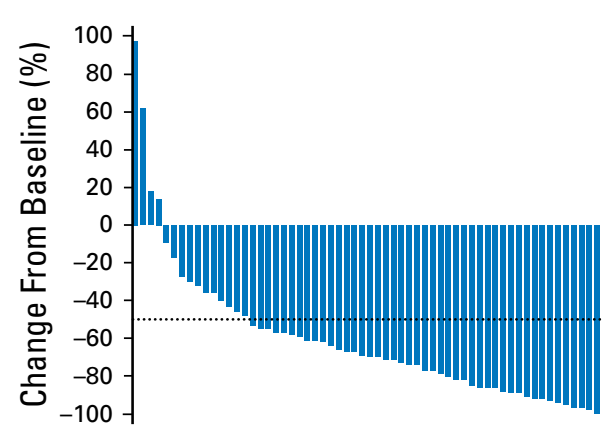

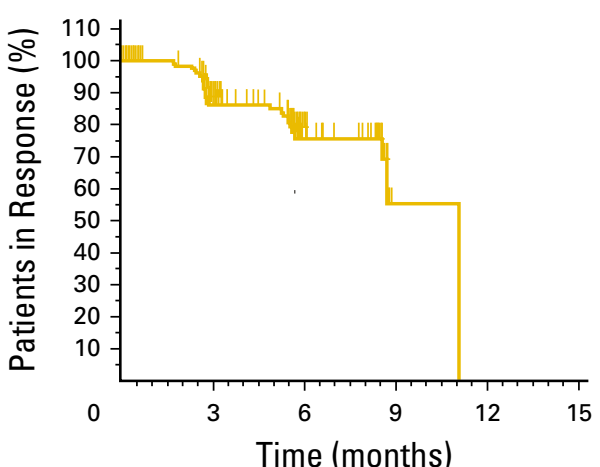

No. at risk $145 \quad 89$

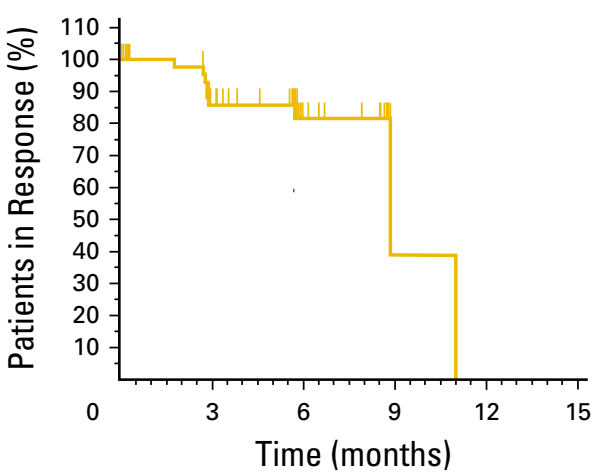
No. at risk
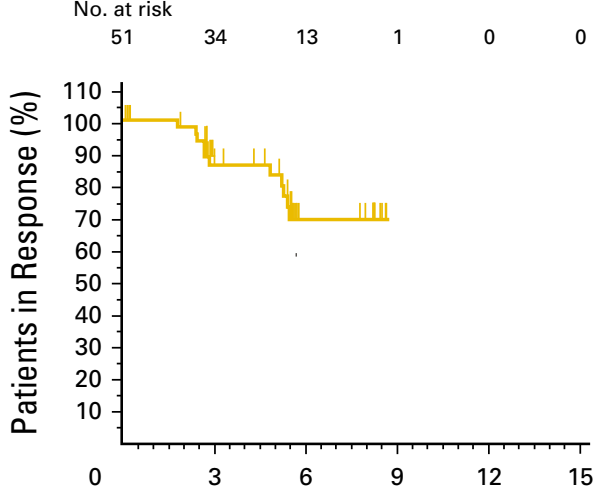

Time (months)

No. at risk
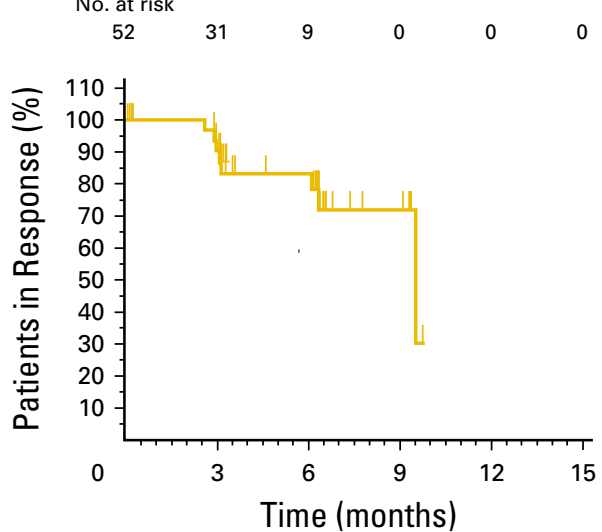

No. at risk
Fig 2. Decrease from baseline in tumor burden (left) and Kaplan-Meier estimates of objective response duration (right) on the basis of central review in patients with response. (A) All cohorts; (B) cohort 1; (C) cohort 2; (D) cohort 3. 
central review, and 176 samples tested positive for PD-L1. Figure 3 shows the distribution of the three PD-L1 expression scores (tumor cell staining intensity, membrane staining of tumor cells, and histiocyte staining) and response to pembrolizumab across all cohorts. Notably, $90.4 \%$ of patients had the highest intensity staining, $88.1 \%$ were $100 \%$ PD-L1 positive by membrane staining, and $71.8 \%$ had maximum PD-L1 positive histiocyte staining. In addition, 114 patients $(64.4 \%)$ had the maximum score across all three PD-L1 expression scores (intensity of 3, histiocyte score of 3, and tumor membrane staining of $100 \%$ ).

\section{Safety}

With a median of 13 treatment cycles, the most common treatment-related AEs (TRAEs) were hypothyroidism (12.4\%) and pyrexia $(10.5 \%)$. The most common grade $3 / 4$ TRAEs were neutropenia (2.4\%), dyspnea (1\%), and diarrhea (1\%; Table 3). Immunemediated AEs (events with potentially drug-related immunologic causes regardless of treatment attribution) and infusion-related reactions were reported in 60 patients $(28.6 \%)$, most commonly hypothyroidism (13.8\%; Data Supplement). Nine patients (4.3\%) discontinued because of TRAEs (myocarditis, myelitis, myositis, pneumonitis, infusion-related reactions, cytokine release syndrome), and 26 patients $(12.4 \%)$ experienced TRAEs resulting in treatment interruptions. Two patients died during follow-up as a result of septic shock and acute graft-versus-host disease, respectively; neither of these deaths were considered to be treatment related.

\section{DISCUSSION}

New therapies are needed for patients with cHL, in particular, for transplantation-ineligible patients and those who relapse after ASCT. In the current study, PD-1 blockade with pembrolizumab demonstrated substantial clinical activity in subsets of heavily pretreated patients with $\mathrm{cHL}$, with most responses observed at the first disease assessment and ongoing at the time of data cutoff. There was a high degree of agreement between response rates per BICR and those per investigator review, both in the whole population and in each cohort. ORRs and CRRs were consistent between the multiple subanalyses of relapsed and refractory patients. This study had two unique patient populations: those with transplantation-ineligible cHL secondary to failure of salvage therapy and BV (cohort 2, 81 of 210; $39 \%$ ) and those with primary refractory disease (73 of $210 ; 35 \%$ ). Notably, high response rates were achieved with pembrolizumab in the chemoresistant population, which is known to have a poor prognosis and few available therapeutic options, and in patients with primary refractory disease. Moreover, the clinical activity of pembrolizumab clearly indicates there is no cross-resistance with cytotoxic agents. Pembrolizumab also demonstrated a high response rate $(71.4 \%)$ in patients who had not previously received BV.

Our results contribute to the increasing evidence of the role of PD-1 inhibition in cHL. The anti-PD-1 antibody nivolumab has shown a high response rate in cHL after failure of both ASCT and BV. ${ }^{18}$ Several aspects of our study were different from the nivolumab study, including the use of a fixed dose of pembrolizumab and administration every 3 weeks versus every 2 weeks with nivolumab. The nivolumab study did not include a separate cohort of patients in which salvage chemotherapy and BV failed and who were ineligible for ASCT (ie, cohort 2 in the current study) and did not report on patients whose disease progressed after ASCT but who were not treated with BV after ASCT (cohort 3 ). In addition, although similar ORRs were reported between the two studies, the CRR by independent central review was higher for pembrolizumab (22\%) compared with nivolumab (9\%). In addition, the safety profile of pembrolizumab in this study was at least comparable with that of nivolumab in patients with rrHL, but with markedly lower frequencies of infusion-related reactions.

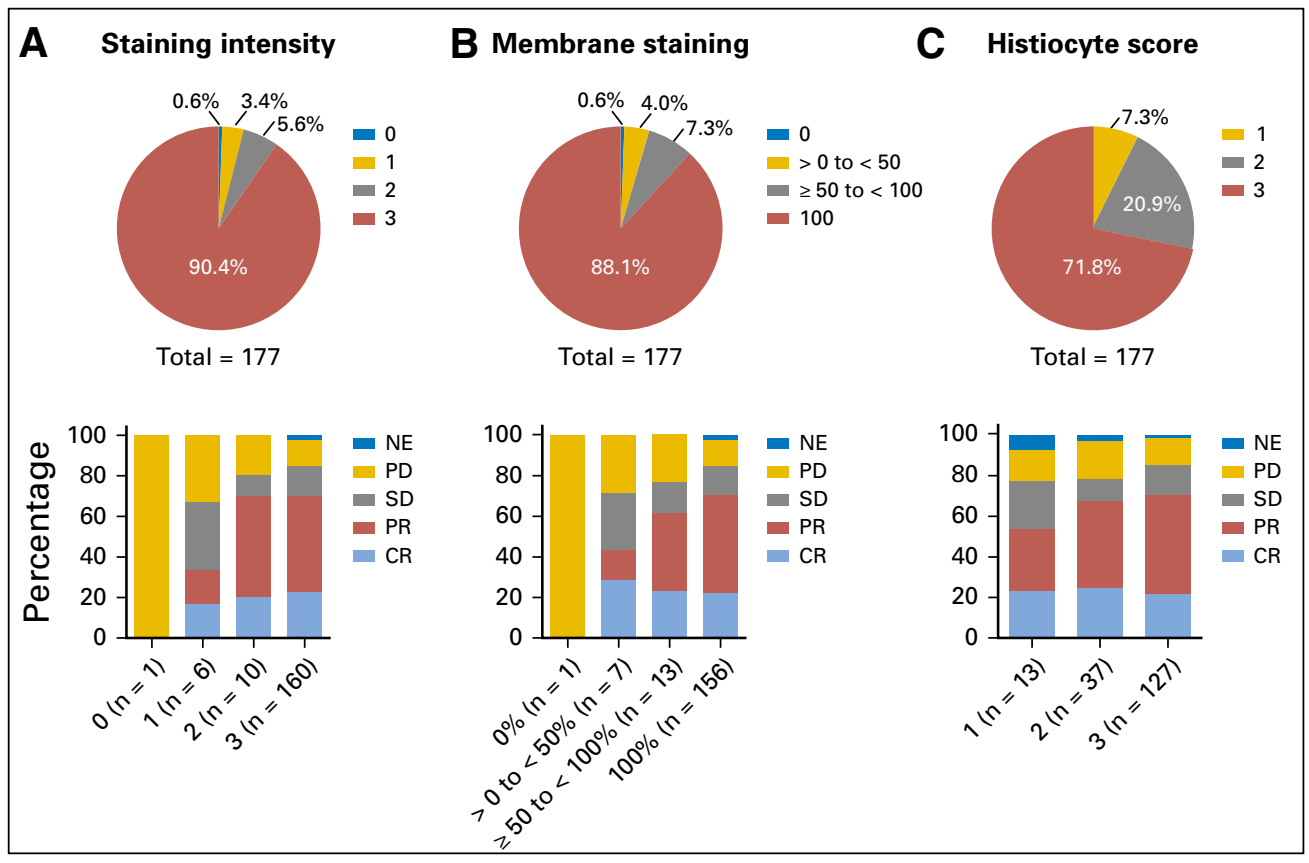

Fig 3. Distribution of programmed death-ligand 1 expression scores and response to pembrolizumab across all cohorts. A total of 177 patients across all cohorts had evaluable pretreatment tumor tissue and best evaluable response per central review. Three scores were reported separately: (A) staining intensity score (0-3); (B) membrane staining score (percentage of tumor cells with membrane staining $0 \%,>0$ to $<50 \%$, $\geq 50$ to $<100 \%$, or $100 \%$ ); and (C) histiocyte score (1-3; semiquantitative assessment of histiocytes/macrophages staining positive for programmed death-ligand 1). CR, complete remission; NE, not estimable; $\mathrm{PD}$, progressive disease; PR, partial response; SD, stable disease. 


\begin{tabular}{|c|c|c|c|c|c|c|}
\hline \multirow[b]{2}{*}{ Adverse Event } & \multicolumn{3}{|c|}{$\begin{array}{c}\text { All-Cause Adverse Events }(\mathrm{N}=210) \\
\text { No. }(\%)\end{array}$} & \multicolumn{3}{|c|}{$\begin{array}{l}\text { Treatment-Related Adverse Events ( } N=210) \\
\text { No. (\%) }\end{array}$} \\
\hline & Grade 1 or 2 & Grade 3 & Grade 4 & Grade 1 or 2 & Grade 3 & Grade 4 \\
\hline Pyrexia & 49 (23.3) & $2(1)$ & 0 & $21(10)$ & $1(0.5)$ & 0 \\
\hline Cough & $44(21)$ & $1(0.5)$ & 0 & $11(5.2)$ & $1(0.5)$ & 0 \\
\hline Fatigue & 40 (19) & $2(1)$ & 0 & $18(8.6)$ & $1(0.5)$ & 0 \\
\hline Diarrhea & $33(15.7)$ & $3(1.4)$ & 0 & $13(6.2)$ & $2(1)$ & 0 \\
\hline Vomiting & 32 (15.2) & 0 & 0 & $8(3.8)$ & 0 & 0 \\
\hline Nausea & 28 (13.3) & 0 & 0 & $12(5.7)$ & 0 & 0 \\
\hline Hypothyroidism & 28 (13.3) & $1(0.5)$ & 0 & 25 (11.9) & $1(0.5)$ & 0 \\
\hline Neutropenia & 7 (3.3) & 4 (1.9) & $2(1)$ & $6(2.9)$ & $5(2.4)$ & 0 \\
\hline Upper respiratory tract infection & 27 (12.9) & 0 & 0 & 7 (3.3) & 0 & 0 \\
\hline Rash & $23(11)$ & 0 & 0 & $16(7.6)$ & 0 & 0 \\
\hline Pruritus & $23(11)$ & 0 & 0 & $8(3.8)$ & 0 & 0 \\
\hline Headache & $22(10.5)$ & $1(0.5)$ & 0 & $13(6.2)$ & 0 & 0 \\
\hline Arthralgia & $21(10)$ & $1(0.5)$ & 0 & $8(3.8)$ & 1 & 0 \\
\hline Constipation & $20(9.5)$ & 0 & 0 & $6(2.9)$ & 0 & 0 \\
\hline Nasopharyngitis & $19(9)$ & 0 & 0 & $2(1)$ & 0 & 0 \\
\hline Dyspnea & $18(8.6)$ & $2(1)$ & 0 & $5(2.4)$ & $2(1)$ & 0 \\
\hline Back pain & $16(7.6)$ & $1(0.5)$ & 0 & $4(1.9)$ & 0 & 0 \\
\hline Oropharyngeal pain & $16(7.6)$ & 0 & 0 & $1(0.5)$ & 0 & 0 \\
\hline Asthenia & $14(6.7)$ & 0 & 0 & $3(1.4)$ & 0 & 0 \\
\hline Myalgia & $14(6.7)$ & 0 & 0 & $5(2.4)$ & 0 & 0 \\
\hline Sinusitis & $13(6.2)$ & 0 & 0 & $1(0.5)$ & 0 & 0 \\
\hline Urinary tract infection & $13(6.2)$ & 0 & 0 & 0 & 0 & 0 \\
\hline Insomnia & $13(6.2)$ & $1(0.5)$ & 0 & $2(1)$ & 0 & 0 \\
\hline Nasal congestion & $13(6.2)$ & 0 & 0 & $3(1.4)$ & 0 & 0 \\
\hline Bronchitis & $12(5.7)$ & $1(0.5)$ & 0 & $2(1)$ & 0 & 0 \\
\hline Chills & $12(5.7)$ & 0 & 0 & $5(2.4)$ & 0 & 0 \\
\hline Anemia & $11(5.2)$ & 8 (3.8) & 0 & $1(0.5)$ & 0 & 0 \\
\hline Muscle spasms & $11(5.2)$ & $1(0.5)$ & 0 & 8 (3.8) & 0 & 0 \\
\hline
\end{tabular}

Responses were seen early with pembrolizumab. Also, fourteen patients in this study went on to receive a stem cell transplantation (10 allogeneic and four autologous). In addition, of the 10 patients who received allogeneic transplantation, one died because of graft-versus-host disease, and the remaining nine are in survival follow-up.

The existing data suggest $200 \mathrm{mg}$ every 3 weeks as the optimal dose for pembrolizumab. This fixed dose is associated with a low rate of discontinuation as a result of AEs and an acceptable safety profile, which differs from the safety profile of current cytotoxic therapies for $\mathrm{CHL}$ and $\mathrm{BV}$.

Because of the known genetic alterations in the PD-L1 pathway in patients with $\mathrm{CHL},{ }^{11,12} \mathrm{PD}-\mathrm{L} 1$ positivity was not a requirement for enrollment in this study. However, the majority of patients in this study were PD-L1 positive by intensity, membrane staining, and histiocyte score. Clinical activity was seen across all groups, including the minority of patients with low expression.

Study limitations include the short duration of follow-up, which precluded the accurate estimation of OS and PFS. The patients in this study are being followed up to assess the durability of response.

Overall, pembrolizumab showed excellent results in both relapsed and refractory patients and was well tolerated at a fixed dose, consistent with prior pembrolizumab clinical experience in oncology patients. A randomized phase III study to compare pembrolizumab with $\mathrm{BV}$ in patients with rrHL has been initiated (ClinicalTrials.gov identifier, NCT02684292).

\section{AUTHORS' DISCLOSURES OF POTENTIAL CONFLICTS OF INTEREST}

Disclosures provided by the authors are available with this article at jco.org.

\section{AUTHOR CONTRIBUTIONS}

Conception and design: Robert Chen, Philippe Armand, Bastian von Tresckow, Alejandro D. Ricart, Arun Balakumaran, Craig H. Moskowitz Collection and assembly of data: Robert Chen, Pier Luigi Zinzani, Philippe Armand, Nathalie A. Johnson, Pauline Brice, John Radford, Vincent Ribrag, Daniel Molin, Theodoros P. Vassilakopoulos, Akihiro Tomita, Bastian von Tresckow, Alejandro D. Ricart, Arun Balakumaran, Craig H. Moskowitz

Administrative support: Bastian von Tresckow, Alejandro D. Ricart, Arun Balakumaran

Provision of study materials or patients: Michelle A. Fanale, Pauline Brice, John Radford, Theodoros P. Vassilakopoulos, Bastian von Tresckow, Alejandro D. Ricart

Data analysis and interpretation: Robert Chen, Michelle A. Fanale, Philippe Armand, Vincent Ribrag, Theodoros P. Vassilakopoulos, Bastian von Tresckow, Margaret A. Shipp, Yinghua Zhang, Alejandro D. Ricart, Arun Balakumaran, Craig H. Moskowitz

Manuscript writing: All authors

Final approval of manuscript: All authors

Accountable for all aspects of the work: All authors 


\section{REFERENCES}

1. Engert A, Raemaekers J: Treatment of earlystage Hodgkin lymphoma. Semin Hematol 53: 165-170, 2016

2. Vassilakopoulos $T P$, Johnson $P W$ : Treatment of advanced-stage Hodgkin lymphoma. Semin Hematol 53:171-179, 2016

3. Kuruvilla J: Standard therapy of advanced Hodgkin lymphoma. Hematology (Am Soc Hemato Educ Program) 2009:497-506, 2009

4. Kuruvilla J, Keating A, Crump M: How I treat relapsed and refractory Hodgkin lymphoma. Blood 117:4208-4217, 2011

5. von Tresckow B, Moskowitz CH: Treatment of relapsed and refractory Hodgkin Lymphoma. Semin Hematol 53:180-185, 2016

6. Moskowitz $\mathrm{CH}$, Nademanee A, Masszi T, et al: Brentuximab vedotin as consolidation therapy after autologous stem-cell transplantation in patients with Hodgkin's lymphoma at risk of relapse or progression (AETHERA): A randomised, double-blind, placebocontrolled, phase 3 trial. Lancet 385:1853-1862, 2015
7. Younes A, Gopal AK, Smith SE, et al: Results of a pivotal phase II study of brentuximab vedotin for patients with relapsed or refractory Hodgkin's lymphoma. J Clin Oncol 30:2183-2189, 2012

8. Forero-Torres $A$, Fanale $M$, Advani $R$, et al: Brentuximab vedotin in transplant-naive patients with relapsed or refractory Hodgkin lymphoma: Analysis of two phase I studies. Oncologist 17:1073-1080, 2012

9. Küppers R: The biology of Hodgkin's lymphoma. Nat Rev Cancer 9:15-27, 2009

10. Mathas S, Hartmann S, Küppers R: Hodgkin lymphoma: Pathology and biology. Semin Hematol 53:139-147, 2016

11. Roemer $M G$, Advani $R H$, Ligon $A H$, et al: $P D$ L1 and PD-L2 genetic alterations define classical Hodgkin lymphoma and predict outcome. J Clin Oncol 34:2690-2697, 2016

12. Green MR, Rodig $S$, Juszczynski $P$, et al: Constitutive AP-1 activity and EBV infection induce PD-L1 in Hodgkin lymphomas and posttransplant lymphoproliferative disorders: Implications for targeted therapy. Clin Cancer Res 18:1611-1618, 2012
13. Keytruda [package insert]. Whitehouse Station, NJ: Merck, 2017

14. Merck Sharp \& Dohme: Keytruda summary of product characteristics. http://www.ema.europa. eu/docs/en_GB/document_library/EPAR___Product_ Information/human/003820/WC500190990.pdf

15. Chatterjee M, Turner DC, Felip E, et al: Systematic evaluation of pembrolizumab dosing in patients with advanced non-small-cell lung cancer. Ann Oncol 27:1291-1298, 2016

16. Armand $P$, Shipp MA, Ribrag $V$, et al: Programmed death-1 blockade with pembrolizumab in patients with classical Hodgkin lymphoma after brentuximab vedotin failure. J Clin Oncol 34: 3733-3739, 2016

17. Cheson BD, Pfistner $B$, Juweid $M E$, et al: Revised response criteria for malignant lymphoma. J Clin Oncol 25:579-586, 2007

18. Younes A, Santoro A, Shipp M, et al: Nivolumab for classical Hodgkin's lymphoma after failure of both autologous stem-cell transplantation and brentuximab vedotin: A multicentre, multicohort, single-arm phase 2 trial. Lancet Oncol 17: 1283-1294, 2016

\section{Affiliations}

Robert Chen, City of Hope National Medical Center, Duarte, CA; Pier Luigi Zinzani, University of Bologna, Bologna, Italy; Michelle A. Fanale, The University of Texas MD Anderson Cancer Center, Houston, TX; Philippe Armand and Margaret A. Shipp, Dana-Farber Cancer Institute, Boston, MA; Nathalie A. Johnson, Jewish General Hospital, Montreal, Canada; Pauline Brice, Hôpital Saint-Louis, Paris; Vincent Ribrag, Institut Gustave Roussy, Villejuif, France; John Radford, The University of Manchester and Christie NHS Foundation Trust, Manchester Academic Health Science Centre, Manchester, United Kingdom; Daniel Molin, Uppsala University, Uppsala, Sweden; Theodoros P. Vassilakopoulos, National and Kapodistrian University of Athens, Laikon General Hospital, Athens, Greece; Akihiro Tomita, Nagoya University Graduate School of Medicine, Nagoya, Japan; Bastian von Tresckow, University Hospital Cologne, Cologne, Germany; Yinghua Zhang, Alejandro D. Ricart, and Arun Balakumaran, Merck, Kenilworth, NJ; Craig H. Moskowitz, Memorial Sloan Kettering Cancer Center, New York, NY.

Supported by Merck, Kenilworth, NJ.

\section{Support}

\section{Prior Presentation}

Presented in part at the ASCO Annual Meeting, Chicago, IL, June 3-7, 2016; European Hematology Association Annual Meeting, Copenhagen, Denmark, June 9-12, 2016; Pan Pacific Lymphoma Conference, Koloa, Hawaii, July 18-22, 2016; 10th International Symposium on Hodgkin Lymphoma, Cologne, Germany, October 22-25, 2016; American Society of Hematology Annual Meeting, San Diego, CA, December 3-6, 2016. 


\section{AUTHORS' DISCLOSURES OF POTENTIAL CONFLICTS OF INTEREST}

Phase II Study of the Efficacy and Safety of Pembrolizumab for Relapsed/Refractory Classic Hodgkin Lymphoma

The following represents disclosure information provided by authors of this manuscript. All relationships are considered compensated. Relationships are self-held unless noted. I = Immediate Family Member, Inst = My Institution. Relationships may not relate to the subject matter of this manuscript. For more information about ASCO's conflict of interest policy, please refer to www.asco.org/rwc or ascopubs.org/jco/site/ifc.

\section{Robert Chen}

Honoraria: Seattle Genetics, Merck, Genentech, Millennium, Pharmacyclics

Consulting or Advisory Role: Seattle Genetics, Merck, Genentech Speakers' Bureau: Seattle Genetics, Genentech, Millennium Research Funding: Seattle Genetics (Inst), Pharmacyclics (Inst), Millennium (Inst)

Travel, Accommodations, Expenses: Seattle Genetics, Genentech, Merck

Pier Luigi Zinzani

Consulting or Advisory Role: Merck, Gilead, Janssen, Roche, Bristol-Myers Squibb, Takeda, Servier, AbbVie

Honoraria: Gilead, Janssen, Merck, Bristol-Myers Squibb, Takeda, Servier, AbbVie, Roche

Michelle A. Fanale

Honoraria: Seattle Genetics, Takeda, Research to Practice, Plexus, BristolMyers Squibb

Consulting or Advisory Role: Amgen, Merck, Bristol-Myers Squibb Research Funding: Millennium, Seattle Genetics, Novartis, MedImmune, Celgene, Molecular Templates, Genentech, Gilead Sciences, Bristol-Myers Squibb, Merck, Kura, ADC Therapeutics, Takeda

Travel, Accommodations, Expenses: Takeda, Spectrum Pharmaceuticals, Research to Practice, Plexus, Bristol-Myers Squibb, Merck

\section{Philippe Armand}

Consulting or Advisory Role: Bristol-Myers Squibb, Merck, Infinity Pharmaceuticals

Research Funding: Bristol-Myers Squibb (Inst), Merck (Inst), Pfizer (Inst), Affimed Therapeutics (Inst), Sequenta (Inst), Otsuka (Inst), Sigma Tau (Inst), Roche (Inst), Tensha Therapeutics (Inst)

Nathalie A. Johnson

Consulting or Advisory Role: Merck, Roche Canada, AbbVie Research Funding: AbbVie

Travel, Accommodations, Expenses: Roche Canada, Merck, AbbVie

\section{Pauline Brice}

Research Funding: Takeda Millennium (Inst), Merck Sharp \& Dohme (Inst), Gilead Sciences (Inst), Bristol-Myers Squibb

\section{John Radford}

Stock or Other Ownership: AstraZeneca (I), GlaxoSmithKline (I)

Consulting or Advisory Role: Takeda, Seattle Genetics, Novartis

Speakers' Bureau: Takeda, Bristol-Myers Squibb

Research Funding: Takeda

Expert Testimony: Takeda

Travel, Accommodations, Expenses: Takeda

\section{Vincent Ribrag}

Honoraria: Infinity Pharmaceuticals, Bristol-Myers Squibb, Eisai, PharmaMar, Gilead Sciences

Consulting or Advisory Role: Infinity Pharmaceuticals, Bristol-Myers Squibb, PharmaMar, Gilead Sciences, NanoString Technologies, Incyte, Merck Sharp \& Dohme

Research Funding: BAY1000394 studies on MCL

Expert Testimony: Servier

Travel, Accommodations, Expenses: Roche, Bristol-Myers Squibb

Daniel Molin

Honoraria: Roche, Merck, Bristol-Myers Squibb, Takeda

Theodoros P. Vassilakopoulos

Honoraria: Roche, Takeda, Bristol-Myers Squibb, Novartis, Genesis Pharmaceuticals

Consulting or Advisory Role: Roche, Takeda, Bristol-Myers Squibb, Novartis, Genesis Pharmaceuticals

Travel, Accommodations, Expenses: Roche, Takeda, Bristol-Myers Squibb, Novartis, Genesis Pharmaceuticals

\section{Akihiro Tomita}

No relationship to disclose

\section{Bastian von Tresckow}

Honoraria: Novartis, Takeda

Consulting or Advisory Role: Novartis, Takeda

Research Funding: Novartis, Merck Sharp \& Dohme, Takeda

Travel, Accommodations, Expenses: Bristol-Myers Squibb

Margaret A. Shipp

Honoraria: Bristol-Myers Squibb, Merck, Gilead Sciences, Takeda Consulting or Advisory Role: Bristol-Myers Squibb, Merck, Gilead Sciences, Takeda

Research Funding: Bristol-Myers Squibb (Inst), Bayer (Inst)

Yinghua Zhang

Employment: Merck

Alejandro D. Ricart

Employment: Merck, Novartis

Stock or Other Ownership: Pfizer, Seattle Genetics

Arun Balakumaran

Employment: Merck

Stock or Other Ownership: Merck

Travel, Accommodations, Expenses: Merck

Craig H. Moskowitz

Consulting or Advisory Role: Merck

Research Funding: Merck, Seattle Genetics, Pharmacyclics

Other Relationship: Celgene, Genentech BioOncology, Merck, Seattle Genetics 


\section{Acknowledgment}

We thank the patients and their families and caregivers for participating in the study; QualTek Molecular Laboratories (Newtown, PA) for programmed death-ligand 1 immunohistochemistry assay testing; Roger Dansey (Merck, Kenilworth, NJ) for critical review of the manuscript; Seth Thompson (Merck) for statistical support; Jonathan Juco and Eric Gustafson (Merck) for biomarker analysis; Thao Vo (Merck) for patient-reported outcomes research; Shelonitda Rose for study design support; and Victoria Fox, Kathryn Fogarty, and Laura Sharon (Merck) for study team support. Medical writing and editorial assistance, funded by Merck, were provided by Sarah Adai, Doyel Mitra, and Matthew Grzywacz, of the ApotheCom oncology team (Yardley, PA). The current affiliation for A.T. is Fujita Health University School of Medicine, Toyoake, Japan. A.D.R. was an employee of Merck, Kenilworth, NJ, at the time of this study. 